\title{
Verborgen im Dreißigjährigen Krieg - der Münzfund von Buchenbach
}

\author{
von Matthias OHM
}

Krisenzeiten sind immer auch Krisenzeiten des Geldes. Wenn staatliche Gemeinwesen Phasen der politischen und wirtschaftlichen Instabilität durchlaufen, lässt sich dies immer auch am Umgang mit dem Geld ablesen. So wird in Zeiten der Not der Edelmetallgehalt der neu geschlagenen Münzen verringert. In Zeiten höchster Not, wenn die Menschen ihr Hab und Gut bedroht sehen, verstecken sie ihr Geld, um es nach Ende der Krise wieder bergen zu können.

Diese beiden Entwicklungen sind überzeitliche Phänomene, die sich im deutschen Südwesten während des 3. wie während des 17. Jahrhunderts ablesen lassen. So geriet das Römische Reich an seinem nördlichen Rand im 3. Jahrhundert immer mehr unter Druck. Mehrmals überrannten germanische Stämme den Limes. Ablesbar ist diese Krise sowohl an den Münzen, deren Silberanteil immer mehr abnahm, als auch an den vielen im 3. Jahrhundert versteckten und nicht mehr geborgenen Schätzen in Südwestdeutschland. ${ }^{1}$

Während des Dreißigjährigen Kriegs, einer weiteren schweren Krisenzeit, sollten sich die Entwicklungen wiederholen. Zu Beginn der 1620er Jahre kam es zur „Kipper- und Wipperzeit““, in der das Geld massiv an Wert verlor. Ein gutes Jahrzehnt später - nach der Niederlage der evangelischen Seite in der Schlacht von Nördlingen - wurde der deutsche Südwesten von katholischen Truppen geplündert und verwüstet. In dieser Zeit versteckten viele Menschen ihren Besitz in der Hoffnung, ihn in friedlichen Zeiten wieder bergen zu können. In vielen Fällen nahmen die Besitzer ihr Geheimnis jedoch mit ins Grab, die verborgenen Schätze blieben jahrhundertelang unentdeckt. ${ }^{2}$

1 Vgl. Gestürmt - geräumt - vergessen? Der Limesfall und das Ende der Römerherrschaft in Südwestdeutschland. Ausstellungskatalog Limesmuseum Aalen 1992, Stuttgart 1992 (Württembergisches Landesmuseum Stuttgart. Führer und Bestandskataloge Archäologische Sammlungen, Bd. 2) und Martin Kemkes: Vom Rhein an den Limes und wieder zurück. Die Besetzungsgeschichte Südwestdeutschlands. In: Imperium Romanum. Roms Provinzen an Neckar, Rhein und Donau. Katalog der Großen Landesausstellung Baden-Württemberg 2005/2006, hg. vom Archäologischen Landesmuseum Baden-Württemberg. Stuttgart 2005, S. 44-53.

2 Vgl. Niklot Klüßendorf: Die Zeit der Kipper und Wipper (1618-1623). Realwert und Nominalwert im Widerstreit. In: Vorträge zur Geldgeschichte im Geldmuseum 2007. Deutsche Bundesbank. Frankfurt 2009, S. 5-38. Matthias Ohm: Geld in der Krise. Im Dreißigjährigen Krieg wurden Münzen entwertet oder vergraben. In: Momente. Beiträge zur Landeskunde von Baden-Württemberg 4/2013, S. 20-23. Matthias Ohm: Der württembergische Hirschgulden. Wirtschafts-, sozial-, geldund literaturgeschichtliche Annäherungen an eine Münze der „Kipper- und Wipperzeit“. In: Rainer 

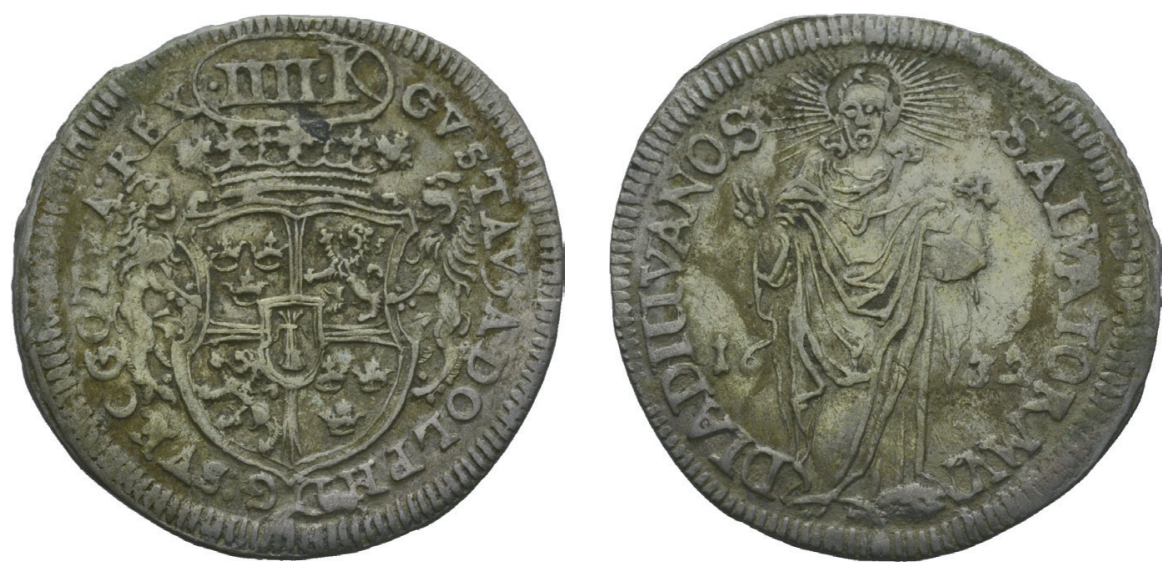

Abb. 1 Im Oktober 1631 konnte der evangelische König Gustav II. Adolf von Schweden (reg. 1611-1632) die katholische Bischofsstadt Würzburg erobern, die rund drei Jahre lang besetzt blieb. Deshalb trägt dieser 1632 in Würzburg geschlagene Batzen auf seiner Vorderseite Namen und Wappen des schwedischen Königs. Die Rückseite zeigt den stehenden Heiland, umgeben von der Inschrift SALVATOR MVNDI ADIUVA NOS - Erlöser der Welt, errette uns!

Nachweis für diese und alle folgenden Abbildungen: Landesmuseum Württemberg, Stuttgart.

Ein Beispiel für einen solchen während des Dreißigjährigen Kriegs versteckten Schatz ist der Münzfund von Buchenbach (im Nordosten des Hohenlohekreises), der 1636 oder etwas später verborgen und erst kurz vor dem Ersten Weltkrieg wieder entdeckt wurde.

\section{Fundumstände}

Der Schatz von Buchenbach wurde im Juni 1913 ,in einem Steinriegel am Kirchberg" gefunden. ${ }^{3}$ Nur wenig später - am 4. Juli 1913 - erwarb das Stuttgarter Münzkabinett die 67 Münzen für 45 Mark. ${ }^{4}$ Für den Finder, den Tagelöhner Johann Salomon, der sicher in einfachsten Verhältnissen lebte, waren die Entdeckung und der anschließende Verkauf der Münzen ein großer Glücksfall.

Albert (Hg.): Böses Geld, schlechtes Geld, falsches Geld. Tagungsband zum 12. Deutschen und 50. Süddeutschen Münzsammlertreffen 2015 in Speyer (Schriftenreihe der Numismatischen Gesellschaft Speyer 53). Speyer 2015, S. 83-102.

3 Peter Goessler: Neue Münzfunde aus Württemberg (1912-1918). In: WVjH NF 28 (1919), S. 24 31, hier S. $27 \mathrm{f}$.

4 Landesmuseum Württemberg, Stuttgart, Münzkabinett, Zugangsbuch Februar 1906-April 1914, Inventarnummer MK 3200, S. 138. 
Um den Wert der 45 Mark, die er für seinen Fund erhielt, einordnen zu können, seien hier einige Preise für Grundnahrungsmittel angegeben: 1913 kosteten 1 Kilogramm Kartoffeln 6 Pfennig, 1 Liter Milch 21 Pfennig, 1 Liter Bier 26 Pfennig und 1 Kilogramm Roggenbrot 30 Pfennig. ${ }^{5}$ Ein ungelernter Eisenbahnarbeiter verdiente in diesem Jahr 23,70 Mark pro Monat, ${ }^{6}$ der vom Stuttgarter Münzkabinett gezahlte Preis entsprach also fast zwei Monatsgehältern eines Eisenbahnarbeiters.

\section{Prägeorte und -jahre, Nominale und Wert der Münzen}

Der Münzschatz von Buchenbach umfasst 67 silberne Münzen, die in verschiedenen Prägestätten geschlagen worden waren. ${ }^{7}$ Im Alten Reich gab es eine Vielzahl geistlicher und weltlicher Herrschaften, die das Recht besaßen, Münzen auszugeben. Auf dem Gebiet des heutigen Bundeslandes Baden-Württemberg
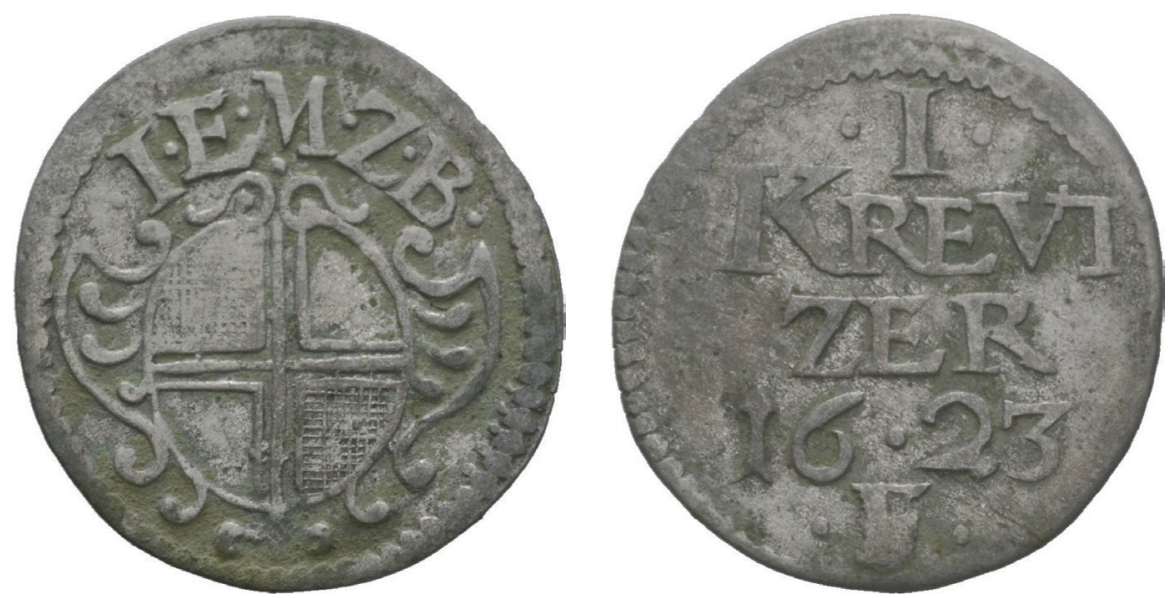

Abb. 2 Auf der Vorderseite dieses Kreuzers findet sich das vierteilige Wappen der Zollern, umgeben von einer Umschrift, die - stark abgekürztNamen und Titel des Münzherrn nennt: I(oachim) E(rnst) M(arkgraf) Z(u) B(randenburg-Ansbach). Die fünfzeilige Inschrift auf der Rückseite gibt das Nominal sowie Prägejahr und-ort an: I KREVTZER 1623 F(ürth).

5 Wolfgang Trapp: Kleines Handbuch der Münzkunde und des Geldwesens in Deutschland, mit 60 Tabellen. Stuttgart 1999, S. 242 f.

6 Ebd., S. 248.

7 Alle Münzen des Fundes von Buchenbach sind im Digitalen Katalog auf der Homepage des Landesmuseums Württemberg abrufbar: <http://www.landesmuseum-stuttgart.de/sammlungen/digitaler-katalog/alle-objekte/>. Die Erfassung des Fundes von Buchenbach wurde durch den Numismatischen Verbund in Baden-Württemberg (NVBW) ermöglicht. 

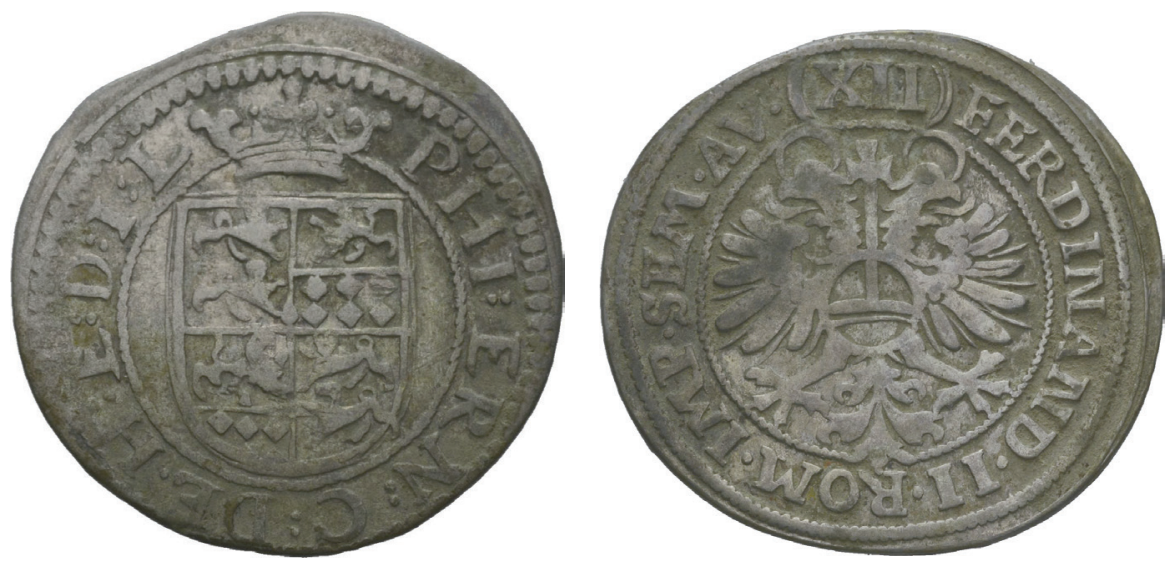

Abb. 3 Der Groschen von Graf Philipp Ernst zu Hohenlohe-Langenburg (reg. 1610-1628) von 1623 zeigt auf dem Avers das Wappen der Grafschaft und auf dem Revers den Doppeladler, der auf seiner Brust die Wertzahl 3 (Kreuzer) trägt. Die Umschriften auf Vorder-und Rückseite nennen Namen sowie Titel des Münzherrn und des Kaisers: PHI(lippus) ERN(estus) C(omes) DE H(ohenlohe) E(t) D(ominus) I(n) L(angenburg) und FERD(inandus) II D(ei gratia) ROM(anorum) Im(perator) SEM(per) AV(gustus).

prägten um die Mitte des 18. Jahrhunderts rund 25 Reichsstände Münzen. ${ }^{8}$ Diese Vielfalt spiegelt sich auch im Fund von Buchenbach wider, in dem Münzen aus 16 Prägestätten vom Elsass bis nach Mähren vorhanden sind. Einen Schwerpunkt nehmen fränkische Münzstätten ein: Rund zwei Drittel der Prägungen stammen aus Franken, aus den Bistümern Bamberg und Würzburg sowie aus den Markgrafschaften Brandenburg-Ansbach und -Bayreuth (Abb. 2, 6, 9, 10).

Zehn Münzen sind rhein-mainische Gemeinschaftsprägungen (Abb. 5); die übrigen Stücke stammen aus Münzstätten der Habsburger, aus schwäbischen und elsässischen Reichsstädten (Abb. 7, 8), aus Bayern und der Pfalz. Schließlich wurde ein Stück auch in der Nähe des Fundorts geschlagen: der Groschen Graf Philipp Ernsts zu Hohenlohe-Langenburg von 1623 (Abb. 3).

Fast alle Münzen des Fundes von Buchenbach stammen aus der Zeit des Dreißigjährigen Kriegs. Lediglich zwei Stücke wurden vor 1618 geprägt: ein Batzen aus der Reichsmünzstätte Nördlingen von 1533 (Abb. 4) und ein Halbbatzen Herzog Reichards von Pfalz-Simmern von 1576. Es kann nur spekuliert werden, ob auch die Münze von 1533 mit dem Bildnis Karls V. wie die übrigen Prägungen nur als Geldstück den Weg in den Schatz fand. Möglicherweise wurde der

8 Ulrich Klein: Die territoriale Gliederung des deutschen Südwestens in napoleonischer Zeit am Beispiel der Münzen. In: Baden und Württemberg im Zeitalter Napoleons. Ausstellungskatalog Württembergisches Landesmuseum Stuttgart 1987. Stuttgart 1987, Bd. 1/1, S. 372 f., hier S. 372. 

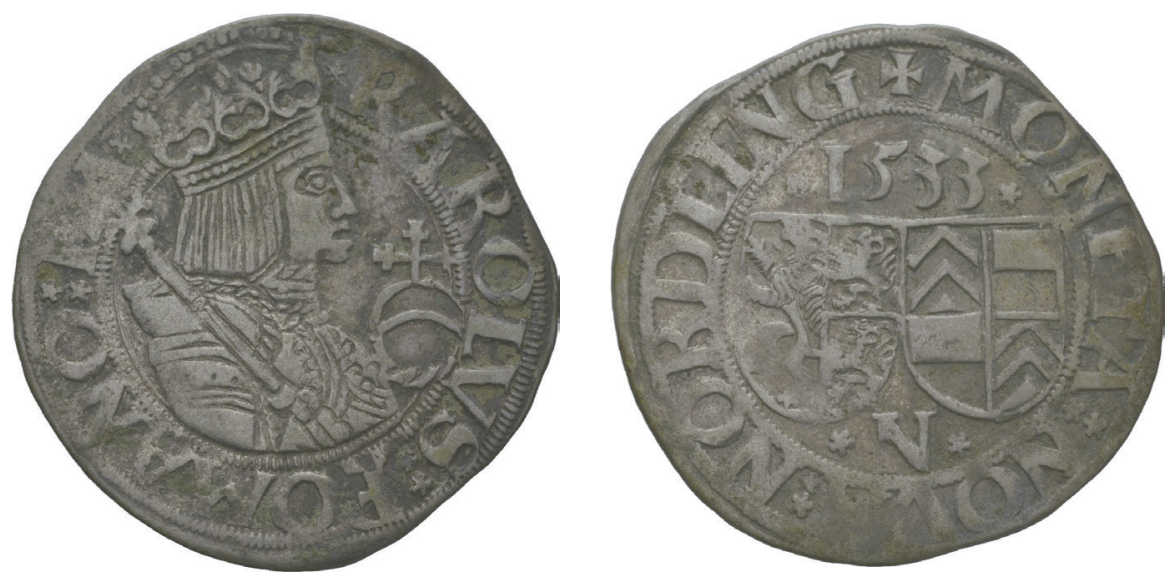

Abb. 4 Die Reichsmünzstätte Nördlingen war im ersten Drittel des 16. Jahrhunderts an Eberhard IV. von Eppstein-Königstein (reg. 1505-1535) verpfändet. Deshalb trägt dieser 1533 in Nördlingen geschlagene Batzen auf dem Revers die Wappen von Eppstein-Minzenberg und Königstein-Dietz. Auf dem Avers ist Karl V. (reg. 1519-1556) im Brustbild nach rechts dargestellt. Der Kaiser trägt eine Krone auf dem Haupt, in seinen Händen hält er Reichsapfel und Zepter. Die Umschrift nennt seinen Titel als römischer Kaiser.

Batzen von 1533 aber auch deshalb aufbewahrt, um die Erinnerung an den Kaiser und den während seiner Regierung geschlossenen Augsburger Religionsfrieden von 1555 wach zu halten - und so an eine Zeit der Verständigung zwischen Katholiken und Evangelischen zu erinnern, die 1618 mit dem Ausbruch des Kriegs (der ein Dreißigjähriger werden sollte) beendet war.

Alle anderen Münzen des Fundes von Buchenbach wurden in den 1620er und 1630er Jahren geprägt. Das jüngste Stück ist ein Halbbatzen aus dem Jahr 1636, den vier rhein-mainische Reichsstände gemeinsam ausgaben (Abb. 5). Diese Prägung ist die so genannte Schlussmünze, die Auskunft über den Verbergungszeitpunkt gibt: Frühestens 1636 kann der Schatz von Buchenbach versteckt worden sein.

Der Münzfund von Buchenbach besteht vor allem aus mittelgroßem Silbergeld. Batzen, Groschen und Halbbatzen - Münzen im Wert von 4, 3 und 2 Kreuzern - machen mit 57 der insgesamt 67 Münzen den bei Weitem größten Anteil aus (Abb 1, 3-6, 9, 10).

Dazu kommen drei große Silberstücke im Wert von 12 bzw. 10 Kreuzern: ein Dreibätzner Graf Johann Reinhards I. von Hanau-Lichtenberg von 1625, ein 12 Kreuzerstück aus der elsässischen Reichsstadt Weissenburg/Wissembourg von 1626 (Abb. 7) sowie ein Zehner Erzherzog Leopolds V. von Tirol aus dem Jahr 1623. 

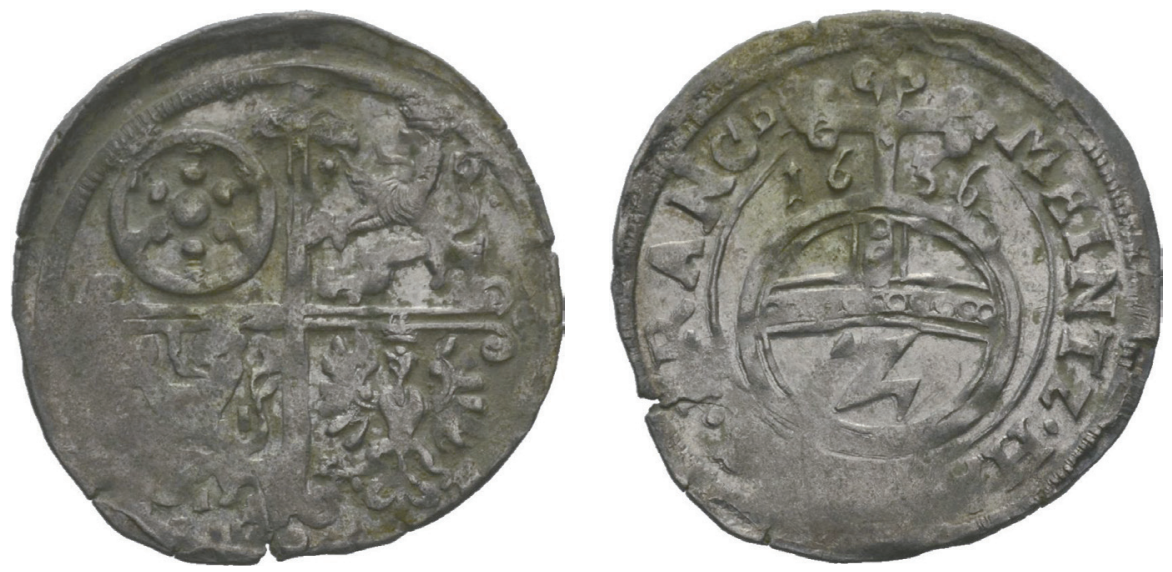

Abb. 5 Die Schlussmünze des Fundes von Buchenbach ist ein Halbbatzen, der 1636 von vier Münzherrschaften im Rhein-Main-Gebiet gemeinsam ausgegeben wurde. Auf dem Avers finden sich ihre Wappen: ein Rad für das Erzbistum Mainz, jeweils ein Löwe für die Landgrafschaft Hessen-Darmstadt und die Grafschaft Nassau-Saarbrücken sowie ein Adler für die Reichsstadt Frankfurt. Der Revers zeigt einen Reichsapfel mit der Wertzahl 2 (Kreuzer), die Umschrift nennt die Namen der vier beteiligten Prägeherrschaften.
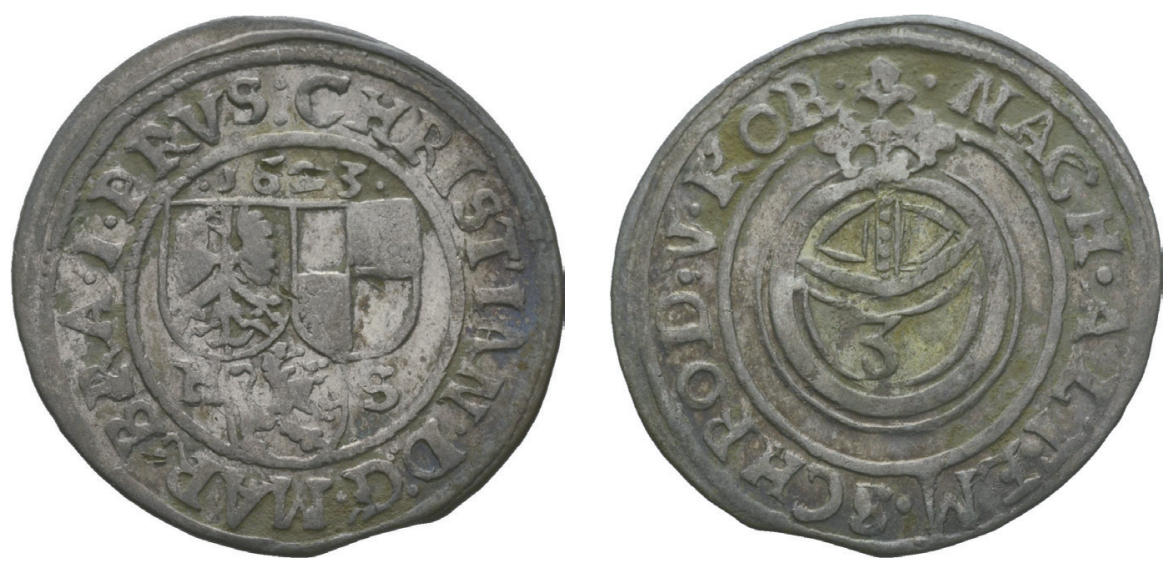

Abb. 6 Der Groschen von Markgraf Christian von Brandenburg-Bayreuth (reg. 1603-1655) aus dem Jahr 1633 zeigt auf dem Avers drei Schilde mit den Wappen der Mark Brandenburg (Adler), der Grafschaft Zollern (geviert) und der Burggrafschaft Nürnberg (Löwe). Auf der Rückseite ist ein Reichsapfel mit der Wertzahl 3 (Kreuzer) dargestellt. 

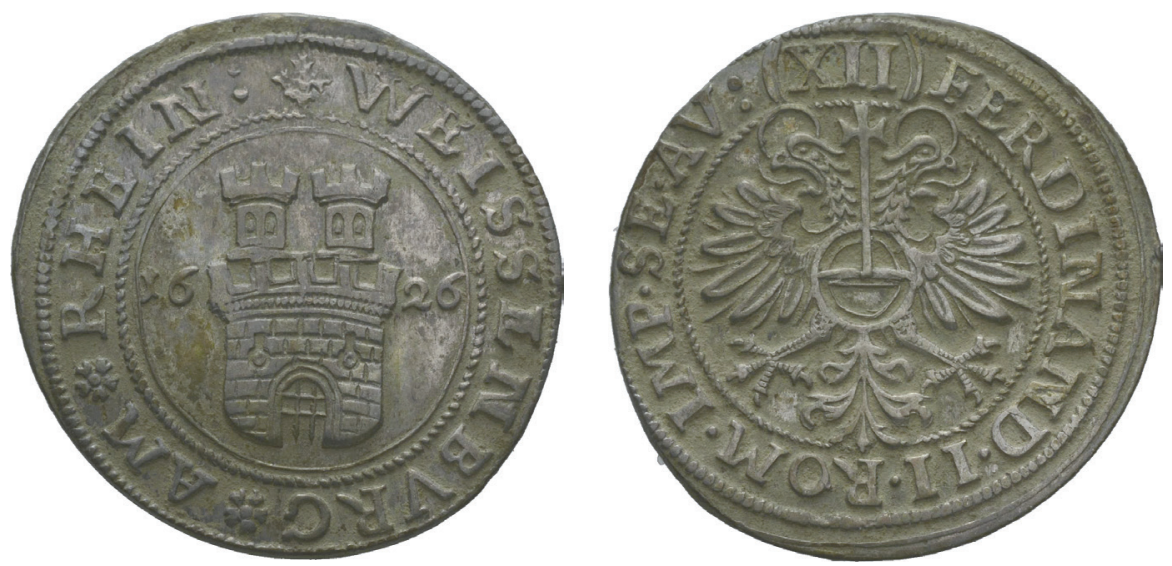

Abb. 7 Das 12-Kreuzerstück aus Weissenburg/Wissembourg von 1626 ist die größte Münze im Fund von Buchenbach. Auf ihrer Vorderseite ist das Wappen der elsässischen Reichsstadt, eine zweitürmige Burg, zu sehen; auf der Rückseite findet sich der doppelköpfige Reichsadler. Die Reversinschrift nennt den Namen des Stadtherrn, Kaiser Ferdinands II. (reg. 1619-1637), und gibt den Wert der Münze an: XII (Kreuzer).
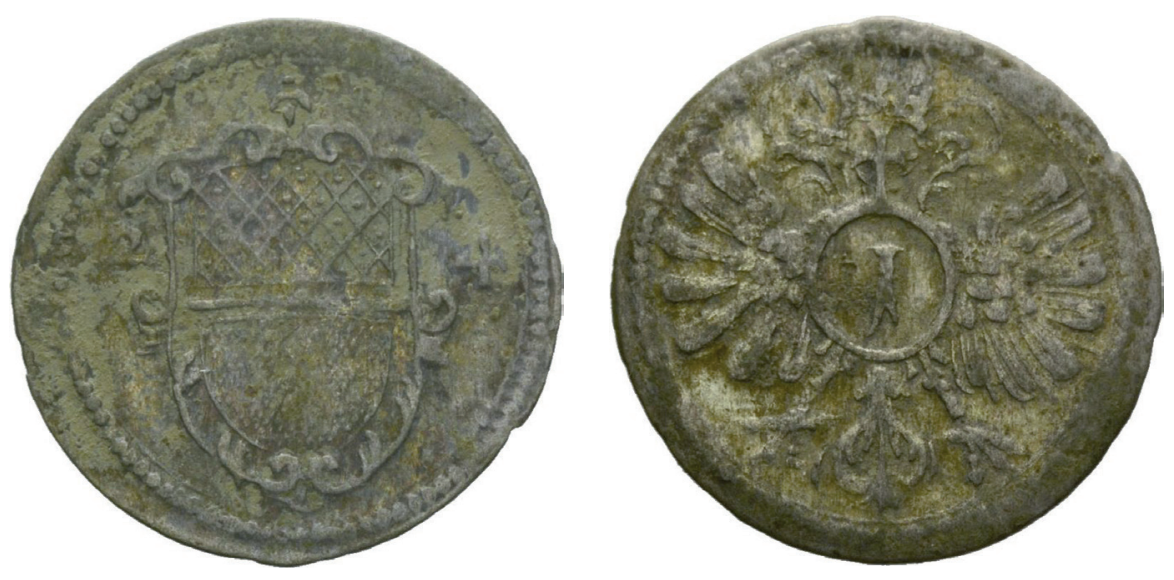

Abb. 8 Der Kreuzer, den die Reichsstadt Ulm 1624 ausgab, zeigt auf der Vorderseite das Wappen der Stadt, den geteilten Schild. Auf der Rückseite ist der Reichsadler dargestellt, dessen Körper von einem Medaillon mit der Wertzahl I (für 1 Kreuzer) gebildet ist. 
An kleinen Silbermünzen schließlich sind sechs 1 Kreuzermünzen (Abb. 2, 8) und ein 2 Pfennigstück, eine Münze im Wert eines halben Kreuzers, vertreten. Im Fund von Buchenbach waren Münzen im Wert von insgesamt 214,5 Kreuzern zusammen getragen. Welchen Wert der Schatz hatte, lässt sich im Vergleich mit Preisen für Grundnahrungsmittel abschätzen. Im Jahr 1636 kostete in Buchenbach ein Ei 1/2 Kreuzer, ein Pfund Brot 1 Kreuzer und ein Pfund Fleisch 5 Kreuzer. ${ }^{9}$

\section{Ein Zeugnis höchster Not}

Der Fund von Buchenbach wurde in höchster Not verborgen. Nach der Schlacht von Nördlingen 1634 wurde Hohenlohe wie der gesamte deutsche Südwesten von plündernden, vergewaltigenden und brandschatzenden Truppen heimgesucht. Auch in den beiden folgenden Jahren, 1635 und 1636, zogen kaiserliche Truppen durch das Hohenloher Land und verbreiteten Angst und Schrecken unter der Bevölkerung, die von Hungersnot und Seuchen ohnehin geschwächt war. ${ }^{10}$ Welches Leid die Menschen erdulden mussten, zeigt ein Ereignis aus dem Jahr 1634, das der damals 13-jährige Sohn des Langenburger Kanzleidirektors überlieferte. Um das vermeintliche Versteck von Münzen zu erfahren, wurde eine Frau zu Tode gefoltert: Die alte Muter im Haus aber haben [...] die Soldaten mit denen [...] Füßen im Schloth aufgezogen, in Meinung Geld zu erpressen, welche jedoch endlich das Leben salvirt. ${ }^{11}$

In einer Zeit solcher Gräueltaten verbarg der ehemalige Besitzer seine 67 Silbermünzen. Wer er war und warum er den Schatz nicht mehr bergen konnte, muss - wie bei jedem Münzfund - offen bleiben: War es ein Landsknecht, der sich aus Franken nach Hohenlohe begeben hatte und dort den Tod fand? War es ein Hohenloher Bauer oder Kaufmann, der Handelskontakte nach Franken hatte? Starb der ehemalige Besitzer an der Pest oder wurde er von umherziehenden Landsknechten erschlagen? Viele weitere Szenarien wären denkbar.

Auch einzelne Münzen aus dem Fund von Buchenbach geben Aufschluss über den Krieg. So repräsentiert die 1632 in Würzburg geprägte Münze die Besetzung von Städten und die Übernahme der Münzstätten durch auswärtige Mächte (Abb. 1).

Einige der in Buchenbach verborgenen Münzen stehen auch für die Überwindung der „Kipper- und Wipperzeit“ (1621-1623), während der in riesigem Umfang unterwertige Münzen in Umlauf gebracht worden waren. In Franken wie im

9 OAB Künzelsau. Stuttgart 1883, ND Magstadt 1968, S. 244.

10 Vgl. ebd., S. 243 ff. und Frank Kleinehagenbrock: Die Grafschaft Hohenlohe im Dreißigjährigen Krieg. Eine erfahrungsgeschichtliche Untersuchung zu Herrschaft und Untertanen, Stuttgart 2003 (VKfgL, Reihe B, 153), insbesondere Kap. III („Kriegserfahrungen hohenlohischer Untertanen").

11 Ebd., S. $127 \mathrm{f}$. 

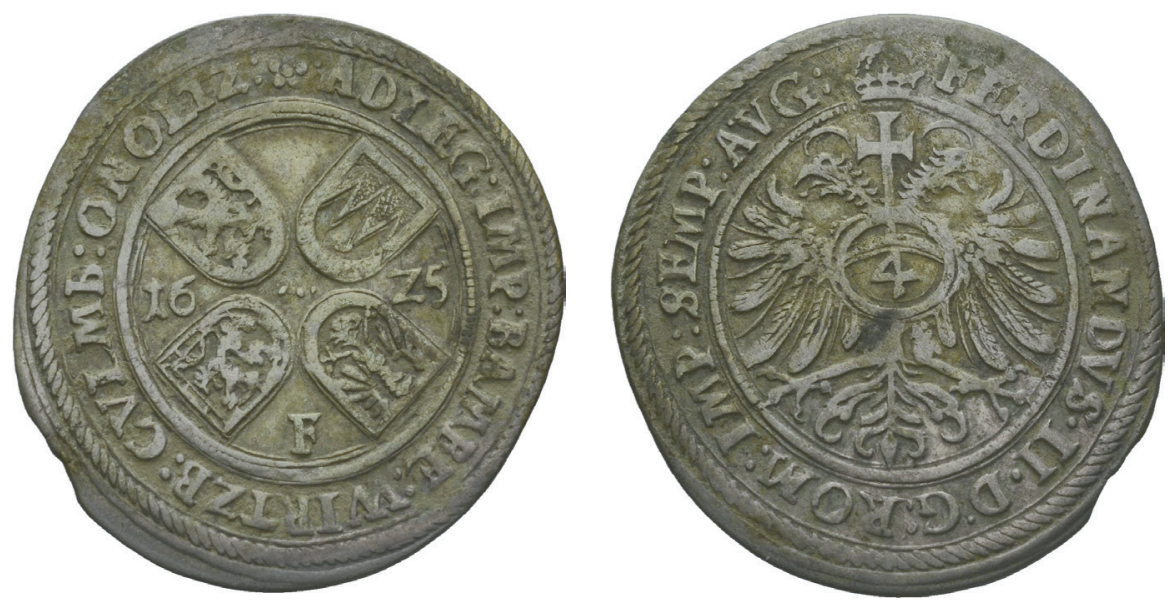

Abb. 9 Im Jahr 1625 gaben die Bischöfe von Würzburg und Bamberg sowie die Markgrafen von Brandenburg-Ansbach und-Bayreuth gemeinsam Münzen aus. Der in Fürth geschlagene Batzen zeigt auf der Vorderseite die Wappen der vier Münzherren und auf der Rückseite den Reichsadler mit der Wertzahl 4 (Kreuzer).

Rhein-Main-Gebiet schlossen sich mehrere Münzherrschaften zusammen, um in einer gemeinsamen Aktion wieder Geld mit dem vorgeschriebenen Silbergehalt auszugeben (Abb. 5, 9). So emittierten die Bischöfe von Bamberg und Würzburg sowie die Markgrafen von Ansbach und Bayreuth ebenso Gemeinschaftsprägungen wie die Erzbischöfe von Mainz, die Landgrafen von Hessen-Darmstadt, die Grafen von Nassau-Saarbrücken und die Reichsstadt Frankfurt, um nach der Krise der „Kipper- und Wipperzeit“ das in Ungleichgewicht geratene Münzwesen wieder in die Balance $\mathrm{zu}$ bringen. ${ }^{12}$

Als Markgraf Joachim Ernst von Brandenburg-Ansbach in den Jahren unmittelbar nach der „Kipper- und Wipperzeit“ Münzen schlagen ließ, betonte er in den Inschriften ausdrücklich, dass die Prägungen den gesetzlichen Bestimmungen des Reiches entsprachen (Abb. 10). Auf diese Weise versuchte der ansbachische Markgraf das Vertrauen in das neu geschlagene Geld zu stärken. Auch der Bayreuther Markgraf Christian wies auf seinem Groschen von 1623 darauf hin, dass die Münze NACH ALTEM SCHROD V(nd) KOR(n) geprägt war, also korrektes Gesamtgewicht und richtigen Edelmetallanteil hatte (Abb. 6).

Der Münzfund von Buchenbach zeigt, welche dramatischen Auswirkungen der Dreißigjährige Krieg auf das Geld und auf den Umgang mit Geld hatte. Der

12 Vgl. Konrad Schneider: Pfennige - Heller - Kupfergeld. Kleingeld im Rheinland vom Spätmittelalter bis ins 19. Jahrhundert. Speyer 2003 (Schriftenreihe der Numismatischen Gesellschaft 43), S. 110 f. und Wolfgang Krug: Die Münzen des Hochstifts Bamberg 1007 bis 1802. Stuttgart 1999 (Süddeutsche Münzkataloge 9), S. 23 f. 

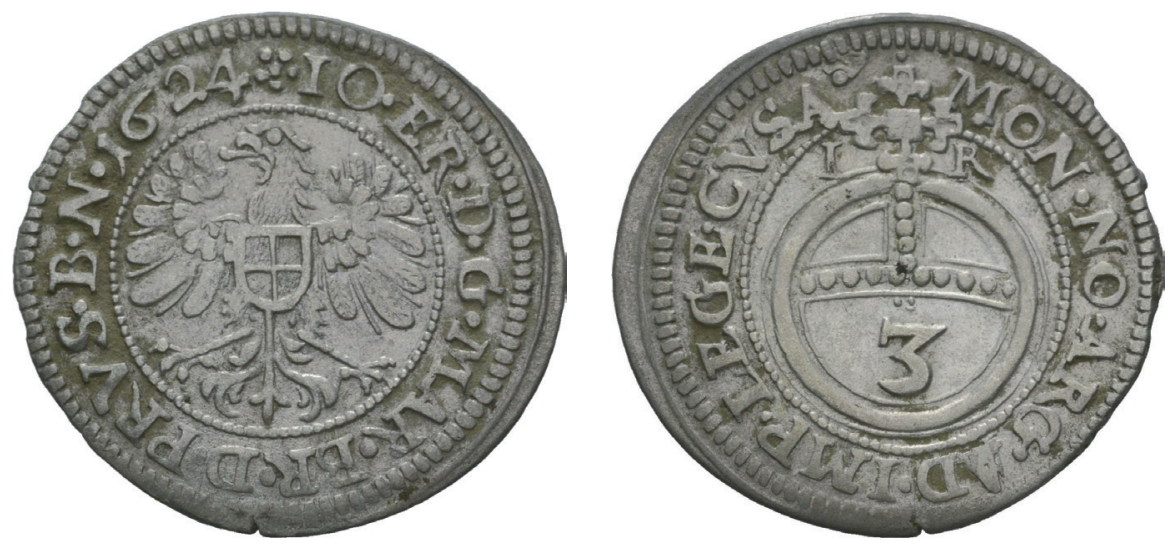

Abb. 10 Der 1625 von Markgraf Joachim Ernst von Brandenburg-Ansbach (reg. 1603-1625) ausgegebene Groschen trägt auf dem Avers den brandenburgischen Adler mit dem zollernschen Wappen auf dem Körper. Der Revers zeigt einen Reichsapfel mit der Wertzahl 3 (Kreuzer), umgeben von der Umschrift MON(eta) NO(va) ARG(entea) AD IMP(erii) LEGE(m) CVSA - neues Silbergeld, geschlagen gemäß dem Gesetz des Reichs.

ehemalige Besitzer verbarg die 67 Silbermünzen, um sie vor Plünderung und Raub zu schützen, nahm sein Geheimnis aber mit ins Grab. Doch nicht nur der Fund als Ganzes ist eine Quelle für das Elend, das der Dreißigjährige Krieg den Menschen im deutschen Süden und Südwesten brachte. Einzelne Münzen des Fundes repräsentieren darüber hinaus die Bemühungen, die Krise des Geldes in der „Kipper- und Wipperzeit“" zu überwinden. 